\title{
Minimization of the Ohmic Loss of Grooved Polarizer Mirrors in High-Power ECRH Systems
}

\author{
D. Wagner ${ }^{1}$ - F. Leuterer ${ }^{1}$ - W. Kasparek ${ }^{2} \cdot$ J. Stober $^{1}$ • \\ ASDEX Upgrade Team ${ }^{1}$
}

Received: 11 August 2016 / Accepted: 7 October 2016 /

Published online: 28 October 2016

C The Author(s) 2016. This article is published with open access at Springerlink.com

\begin{abstract}
A set of two corrugated polarizer mirrors is typically used in high-power electron cyclotron resonance heating $(\mathrm{ECRH})$ systems to provide the required polarization of the ECRH output beam. The ohmic losses of these mirrors can significantly exceed the losses of plane mirrors depending on the polarization of the incident beam with respect to the orientation of the grooves. Since polarizer mirrors incorporated into miter bends of a corrugated waveguide line are limited in size, active water cooling can become critical in highpower cw systems like the one for ITER. The ohmic loss of polarizer mirrors has been investigated experimentally at high power. A strategy to minimize the losses for given mirror geometries has been found.
\end{abstract}

Keywords Electron cyclotron resonance heating $\cdot$ Polarizer $\cdot$ Ohmic loss

\section{Introduction}

In modern high-power electron cyclotron resonance heating $(\mathrm{ECRH})$ systems, the power transmission capability may be limited by high losses in some components of the transmission line. In corrugated HE11 waveguide lines, one of the critical elements are polarizer mirrors inserted into miter bends. Typically, a set of two corrugated polarizer mirrors is applied to provide the required polarization (usual elliptical) of the ECRH beam injected into the plasma. For single frequency systems, it usually consists of an elliptical and a linear polarizer with

D. Wagner

dietmar.wagner@ipp.mpg.de

ASDEX Upgrade Team

1 Max-Planck-Institut für Plasmaphysik, Boltzmannstr.2, 85748 Garching, Germany

2 Institut für Grenzflächenverfahrenstechnik und Plasmatechnologie, Universität Stuttgart, Pfaffenwaldring 31, 70569 Stuttgart, Germany 
effective groove depths corresponding to $\approx \lambda_{0} / 8$ and $\approx \lambda_{0} / 4$ (universal polarizer) with $\lambda_{0}$ being the free space wavelength [1-3]. Such a universal polarizer can provide any arbitrary polarization in the output beam of the transmission line. In quasi-optical transmission lines, these polarizers can be made large $[4,5]$, but polarizers in miter bends of HE11 transmission lines have a limited diameter and thus a very high incident power density. With increasing power and pulse length, the ohmic loss of the polarizer mirrors cannot be neglected anymore. In particular, for systems like in ITER with high-power beams of $1 \mathrm{MW}$ and up to $3600 \mathrm{~s}$ pulse length, effective cooling of these mirrors can become a serious issue.

The ohmic loss of corrugated polarizer mirrors has been calculated using a 2D FDTD algorithm [6] and space harmonic calculations [2]. Cold tests with grooved polarizer mirrors made out of copper have been performed using a quasi-optical three-mirror resonator [7]. In [2], it was shown that the loss of such mirrors can vary considerably depending on their setting.

We present here high-power tests on similar polarizer mirrors made out of stainless steel which were inserted into an HE11 miter bend. The measurements were performed using a gyrotron of the ASDEX Upgrade (AUG) ECRH system [8] at $140 \mathrm{GHz}$. The measurements with the corrugated polarizer mirrors were cross-calibrated with similar measurements using a plane mirror made of the same material. A simple formalism could be revealed which allows the calculation of the ohmic loss of each polarizer mirror for any given incident polarization and rotation angle. In Chapter 4, we show that there are not more than four possible combinations of the angular settings of the two mirrors of a universal polarizer for any wanted output polarization [9]. Using this formalism, the combination of polarizer angles resulting in the lowest possible ohmic attenuation can be found for each required polarization of the output beam.

\section{Experimental Arrangement}

For the high-power tests, the two polarizer mirrors were fabricated out of stainless steel (VA 1.4311 ) in order to get an easily measurable high temperature difference together with a slow heat transfer towards the mirror edges. The thickness of the test mirrors was $5 \mathrm{~mm}$. The polarizer mirrors have a sinusoidal corrugation with depth 0.8 and $0.57 \mathrm{~mm}$, respectively, and a corrugation period of $1.07 \mathrm{~mm}$. For comparison, a plane disk, also with a thickness of $5 \mathrm{~mm}$ of the same material, was prepared. These mirrors were, each one at a time, inserted into a $90^{\circ}$ miter bend of the AUG transmission line operating in air (Fig. 1). Their diameter is $140 \mathrm{~mm}$ covering the 87-mm inner diameter of the corrugated waveguides. All mirrors under test were equipped with a PT-100 platinum resistance temperature detector at the center of the back side whose dimension is $2 \times 2 \mathrm{~mm}$, thus averaging over two corrugation periods. The sensitivity is $6^{\circ} \mathrm{K} / \mathrm{V}$. At the end of each AUG transmission line, the beam can be directed into a short-pulse calorimetric load via another miter bend with directional coupler.

For the polarizer tests, one of the gyrotrons was operated at $140 \mathrm{GHz}$ into such a calorimetric load. For the tests, we applied an output power of $375 \mathrm{~kW}$ with a pulse length of $50 \mathrm{~ms}$ resulting in easily measureable temperature differences of several degrees. The response time of the PT- 100 detectors on the back side is about $0.5 \mathrm{~s}$. Figure 2 shows a typical temperature signal following a 50-ms gyrotron pulse. The maximum is reached $4 \mathrm{~s}$ after the pulse start. For the central position of the sensor, there is only a very small radial temperature gradient, and therefore, the radial heat transfer is small compared to the heat transfer to the backside, as can be seen in the slow decay of the temperature signal after reaching the maximum compared to the fast rise of the temperature right after the 50-ms gyrotron pulse. 


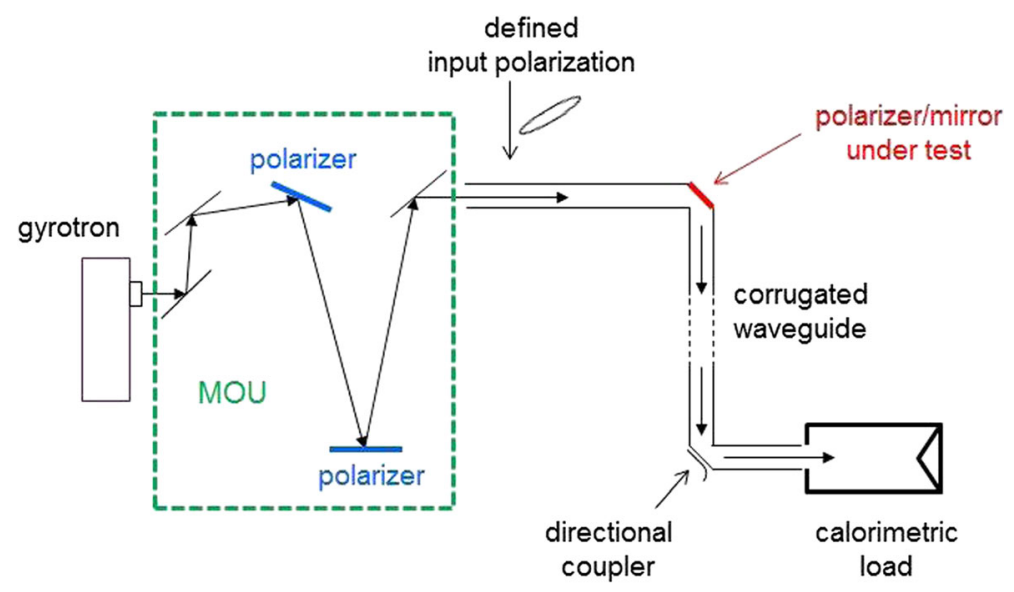

Fig. 1 Measurement setup

The maximum temperature can thus be taken as a measure of the energy deposited on the front mirror surface by the beam. After each pulse, the mirror was cooled back to room temperature by pressurized air. Using the polarizer mirrors in the matching optics unit, any required input polarization onto the miter bend mirror under test can be set.

\section{Test Results}

\subsection{A. Plane Mirror}

In order to calibrate the polarizer losses, a first series of measurements was performed with the plane stainless steel mirror with a linear incident polarization which was rotated from $\varphi=0^{\circ}$ to $\varphi=180^{\circ}$ using the polarizer mirrors in the MOU box. The angle $\varphi$ is defined as the angle of the incident electric field vector with respect to the incidence plane on the miter bend (Fig. 3).

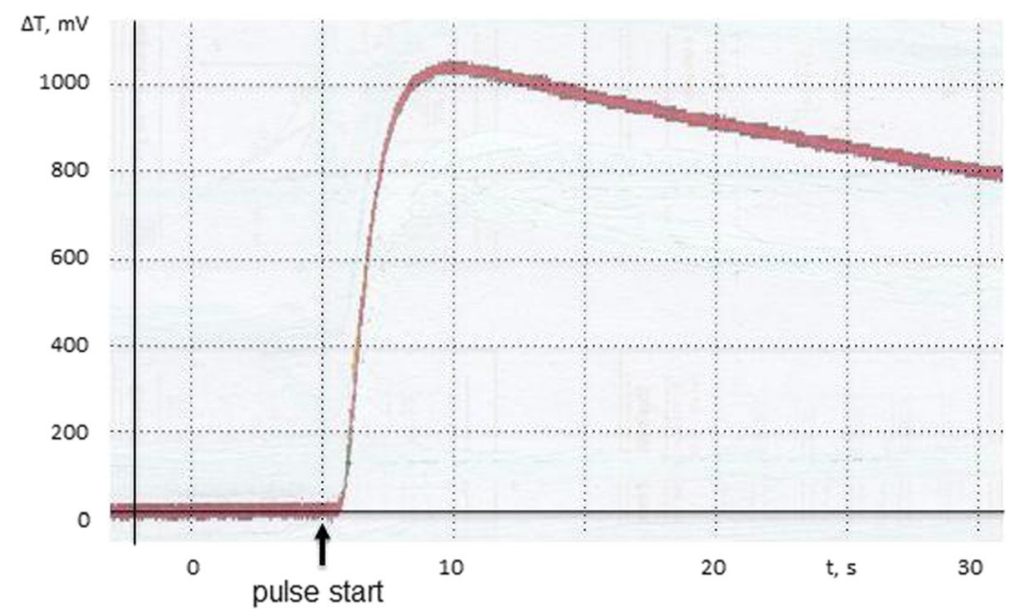

Fig. 2 Measured temperature sequence of the central PT-100 detector at a mirror under test 
angle of incident linear polarization:

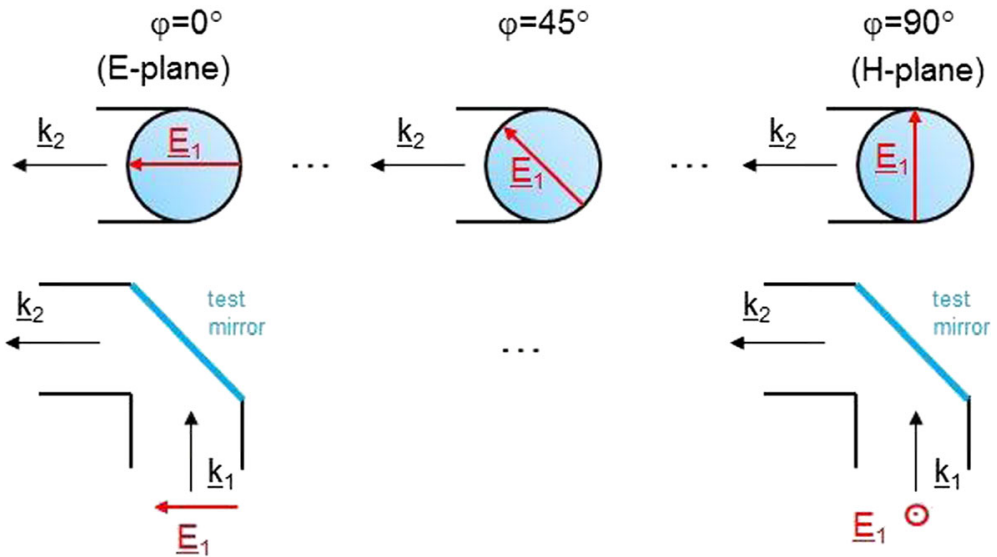

Fig. 3 Definition of the polarization plane with respect to the miter bend mirror (top, front view; bottom, top view)

The result of the measurement is given in Fig. 4. For the data at $\varphi=0^{\circ}$ and $\varphi=90^{\circ}$, we have theoretical results [7] and

$$
\begin{aligned}
& a_{\phi=0^{\circ}}=a_{\perp} / \cos \Theta, \\
& a_{\phi=90^{\circ}}=a_{\perp} \cdot \cos \Theta,
\end{aligned}
$$

where $\Theta$ is the angle of incidence and

$$
a_{\perp}=4 R_{S} / Z_{0} .
$$

The absorption coefficient at perpendicular incidence, $\Theta=0^{\circ}$, on a plane mirror. $R_{\mathrm{S}}$ is the surface resistance, and $Z_{0}$ is the wave impedance.

In our case of a $90^{\circ}$ miter bend, we have $\Theta=45^{\circ}$, and from the two measurements at $\varphi=0^{\circ}$ and $90^{\circ}$, we can determine $a_{\perp}$ to correspond to $a_{\perp m V}=573 \mathrm{mV}$ (respectively, $3.438 \mathrm{~K}$ ). In the following graphs, we normalize the measured $\Delta \mathrm{T}$ values to $a_{\perp m V}$ as

Fig. 4 Ohmic loss of a plane stainless steel mirror in a $90^{\circ}$ miter bend (I.D. $=87 \mathrm{~mm}, \mathrm{f}=140 \mathrm{GHz}$ ). Dots, experimental data; line, model calculation; right scale, data normalized to the case of perpendicular incidence

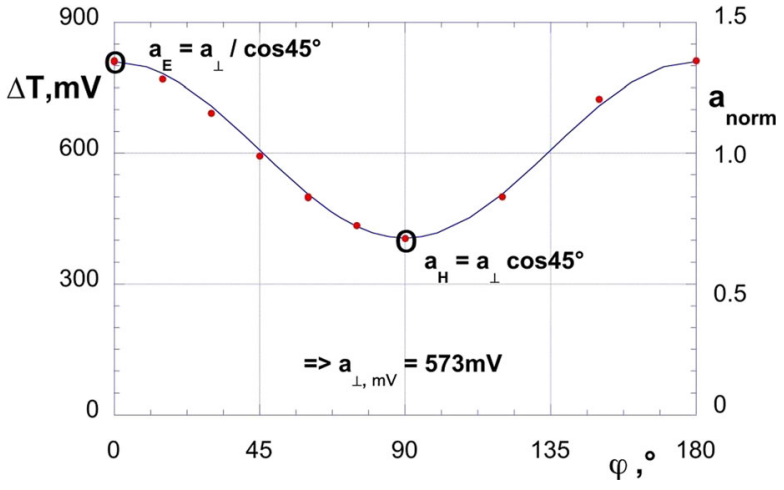




$$
a_{\text {norm }}=\Delta T[m V] / a_{\perp[m V]} .
$$

Neglecting radial diffusion we can determine the absorbed energy $\Delta E_{0}$ per unit area in the center of the mirror of thickness $d$ as

$$
\Delta E_{0}=d \cdot c \cdot \rho \cdot \Delta T
$$

The incident power density $p_{0}$ on the center of the mirror surface can be calculated from

$$
p_{0}=P /\left(R^{2} \pi\right) \cdot f_{p} \cdot \sqrt{2} / 2=165.45 \mathrm{~W} / \mathrm{mm}^{2},
$$

where $P=375 \mathrm{~kW}$ is the total injected power, $2 R=87 \mathrm{~mm}$ is the diameter of the waveguide, $\sqrt{2} / 2$ is due to the $45^{\circ}$ incidence on the mirror, and

$$
f_{p}=\int_{0}^{R} 2 \pi r \cdot d r / \int_{0}^{R} 2 \pi\left(J_{0}(2.405 r / R)\right)^{2} r \cdot d r=0.5 / \int_{0}^{1}\left(J_{0}(2.405 r)\right)^{2} r \cdot d r=3.711(16)
$$

is the peaking factor for a HE11 wave. The energy $\Delta E_{0}$ per unit area absorbed in the center of the mirror during a pulse of $\tau=50 \mathrm{~ms}$ is then

$$
\Delta E_{0}=a \cdot p_{0} \cdot \tau
$$

Together with Eq. (4), and using the data for stainless steel, heat capacity $c=0.5 \mathrm{~J} /(\mathrm{g} \cdot \mathrm{K})$, specific mass $\rho=7.9 \mathrm{~g} / \mathrm{cm}^{3}$, and the value of $\Delta T=3.438 \mathrm{~K}$ corresponding to $a_{\perp}$, we can estimate the absorption coefficient as $a_{\perp}=0.82 \%$, which gives for $E$-plane polarization $(\varphi=$ $\left.0^{\circ}\right) a_{\mathrm{E}}=1.16 \%$ and for $H$-plane polarization $\left(\varphi=90^{\circ}\right) a_{\mathrm{H}}=0.58 \%$. These estimated values agree reasonably well with theory which gives 0.993 and $0.496 \%$ for $E$-plane and $H$-plane incidence, respectively $[7,10]$. Note that some increase of the ohmic loss due to surface roughness with respect to theory is expected.

\subsection{B. Polarizer Mirrors}

For the following description of the experiments, we introduce two coordinate systems. The first, Fig. 5a, relates to the feeding waveguide with the $z$-axis aligned along the incident $k$ vector of the mirror under test and the $x$-axis lying in the incidence plane. The polarization of an incident linearly polarized wave is given by the angle $\varphi$ of the electric field vector with respect to the $x$-axis. The other coordinate system, Fig. $5 \mathrm{~b}$, relates to the mirror under test with the $x 2-z 2$-plane being the mirror surface, the $x 2$-axis oriented perpendicular to the grooves, and the $z 2$-axis along the grooves. The orientation of the grooved mirror is described by the angle $\alpha$ between the grooves and the incidence plane.

In Fig. 6, we show the measured data (dots) when inserting either the $\lambda / 8$ or the $\lambda /$ 4 mirror or the plane mirror into the miter bend. The lines are model calculations as explained later. The data are normalized to the case of perpendicular incidence on to a plane mirror as described above. The figures show a scan of the angle $\varphi$ of a linear 

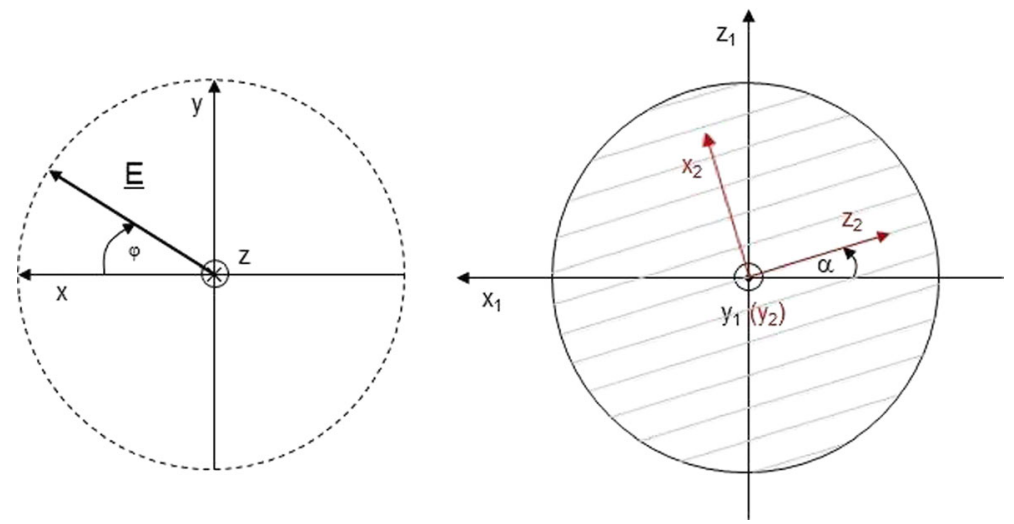

Fig. 5 Definitions of the angles $\varphi$ and $\alpha$ defining polarization and groove orientation. Left, in feeding waveguide; right, on the mirror under test

polarized incident wave, while the orientation $\alpha$ of the grooves of the mirror is constant at either $\alpha=0$ or $90^{\circ}$. In Fig. 7, the graphs show a scan of the groove

Fig. 6 Normalized absorption coefficient as a function of the angle $\varphi$ of a linear polarized incident wave at constant groove orientation angle $\alpha=0^{\circ}$ (top) or $90^{\circ}$ (bottom). Dots, experimental data; lines, model calculation; green, plane mirror; blue, $\lambda / 8$ mirror; red, $\lambda / 4$-mirror
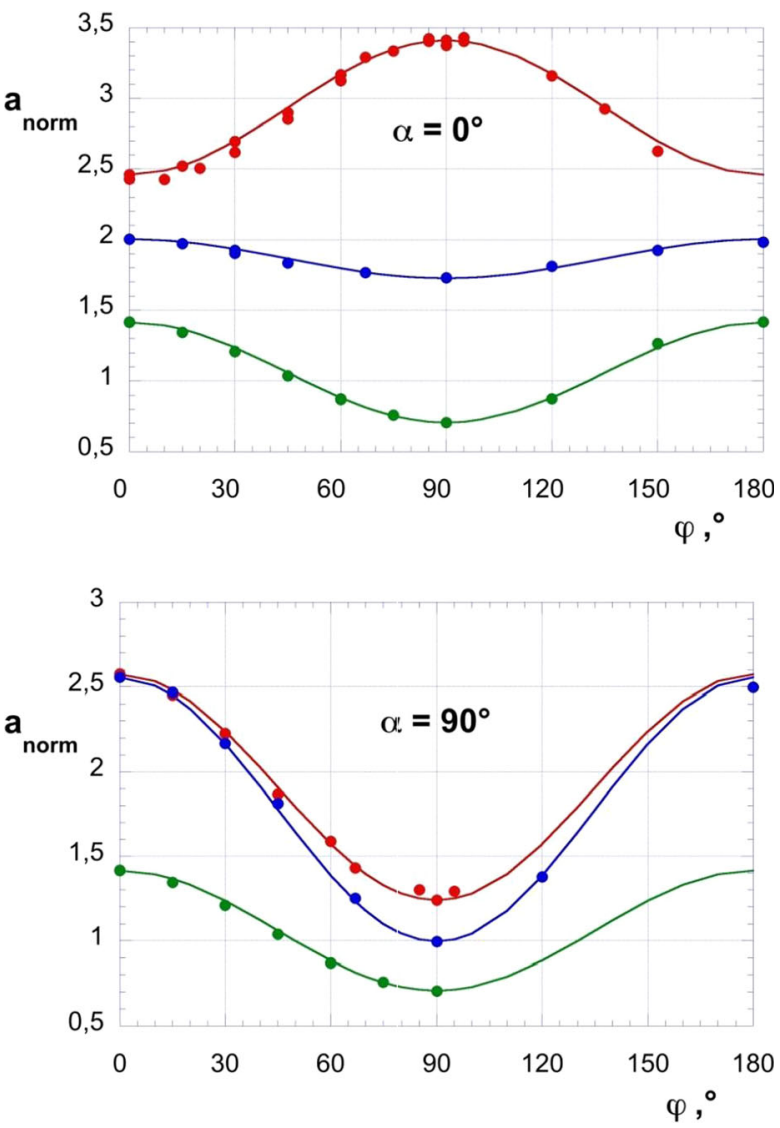
Fig. 7 Normalized absorption coefficient as a function of the groove orientation angle $\alpha$ at constant linear incident polarization angle $\varphi=0^{\circ}$ (red) or $90^{\circ}$ (blue) for the $\lambda / 8$-mirror (top) and the $\lambda / 4$-mirror (bottom)
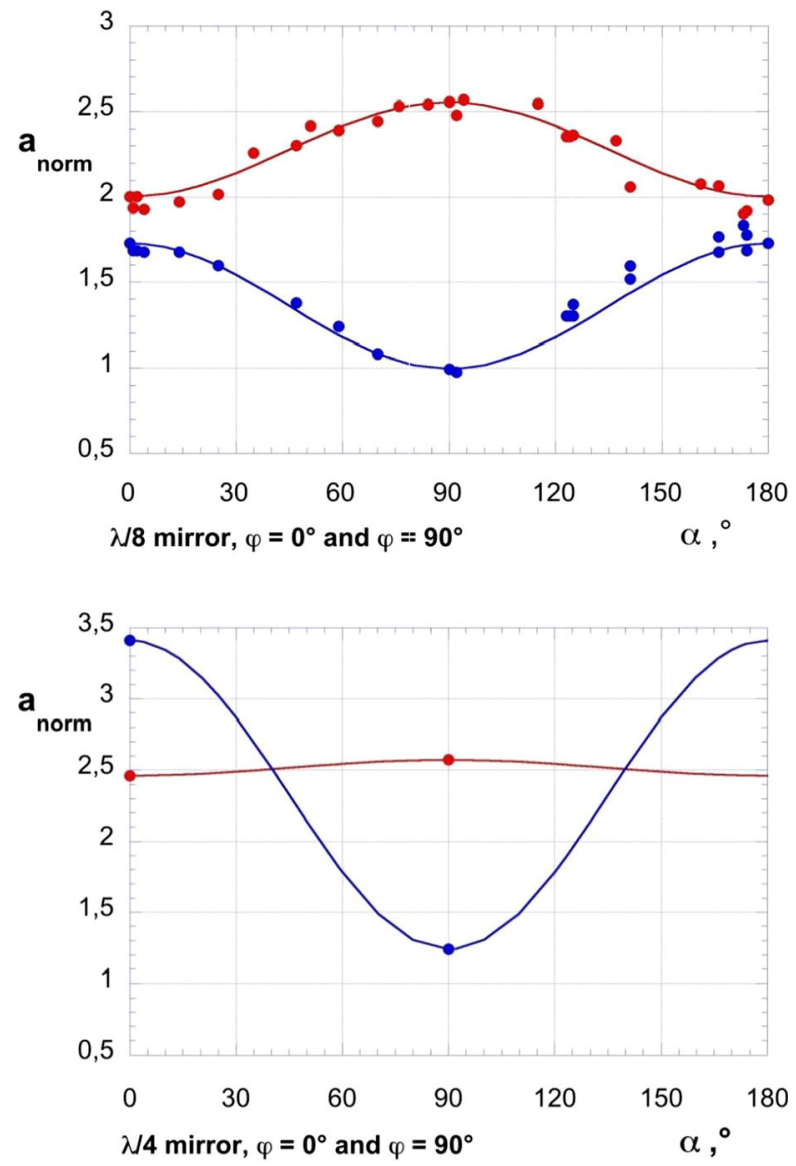

angle $\alpha$, while the angle $\varphi$ of the incident linear polarization is constant at $\varphi=0^{\circ}$ or $90^{\circ}$.

There is no absorption coefficient $a_{\text {norm }}<1$. A prominent feature in all these curves is that they fit to a dependence of $\cos (2 \varphi)$, respectively, $\cos (2 \alpha)$, suggesting a dependence on the square of the incident field components, which scale as $\sin \varphi$ or $\sin \alpha$.

\subsection{Special Cases}

For application to ECRH in fusion experiments, we need in general an elliptical polarization, defined by the ellipticity $\varepsilon$, the orientation of the ellipse $\beta$ with respect to the confining magnetic field, and the sense of rotation of the electric field $r$. To realize this in a waveguide transmission line, a universal polarizer with two polarizer mirrors of $\approx \lambda / 8$ and $\approx \lambda / 4$ groove depth, mounted in $90^{\circ}$ miter bends, i.e., with an incidence angle of $45^{\circ}$, is required. For a single frequency application (in this paper $140 \mathrm{GHz}$ ), there are up to four possible combinations of their groove angles $\alpha$ for each output polarization. Examples are shown in Figs. 12 and 13. Similar results were obtained for broadband polarizers with a steeper incident angle $\left(17.2^{\circ}\right)$ at $140 \mathrm{GHz}$ with groove depths that corresponded to $\lambda / 8$ and $\lambda / 4$ at their design frequency of 122.5 GHz [9]. From the above measurements, we have to expect that these combinations 
Fig. 8 Arrangement of the two polarizer mirrors for the experimental examples and model calculations

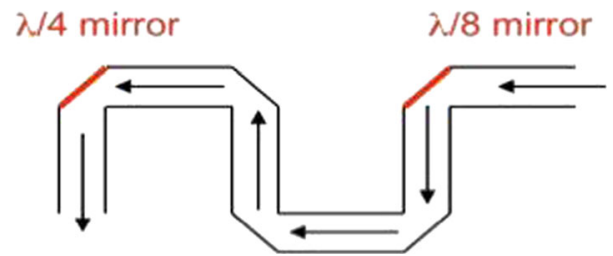

have different losses. In the following, we compare for a few examples for the losses for cases with four possible combinations. To calculate the necessary combinations of mirror angles, we assume the arrangement shown in Fig. 8 where the $\lambda / 8$-mirror is followed by the $\lambda / 4$-mirror. All calculations are performed for incident angles of $45^{\circ}$.

The apparently complicated arrangement in Fig. 8 takes into account that in our present setup, we have only one place where we can insert a rotatable polarizer mirror into a miter bend. Therefore, we proceed in the following way: we calculate the settings required for a two-mirror arrangement as in Fig. 8 with a linear polarized incident field. Then, we insert the $\lambda / 8$ mirror, set it to the calculated orientation $\alpha_{\lambda / 8}$, and determine its normalized loss. Next, we insert the $\lambda / 4$ mirror, set it to the calculated value of $\alpha_{\lambda / 4}$, feed it with an elliptic input polarization corresponding to the calculated output of the $\lambda / 8$ mirror, and determine its loss. This elliptic input polarization can be set by the polarizer mirrors in the MOU box. The total loss is the sum of the individual losses.

In Fig. 9, we see, for example, the case where we want as output a linear polarized field in horizontal direction. The left figure is obtained with a linear polarized incident field in $\varphi=0^{\circ}$ direction ( $E$-plane incidence), and the right figure with an incident field in $\varphi=90^{\circ}$ direction ( $H$-plane incidence). The four possible settings in the first case give absorption coefficients in the range 4.2 to 5.4. In the second case, the absorption coefficients vary between 2.2 and 5 . In order to verify that we get the proper output polarization, we also recorded the detector signal obtained at the directional coupler at the end of the transmission line (Fig. 1) which must be constant in all eight cases.

In Fig. 10, we show an example for an elliptical output polarization with $\varepsilon=0.418$, $\beta=76.4^{\circ}$, and $r=-1$, again with a linear input polarization either horizontal or vertical. Here
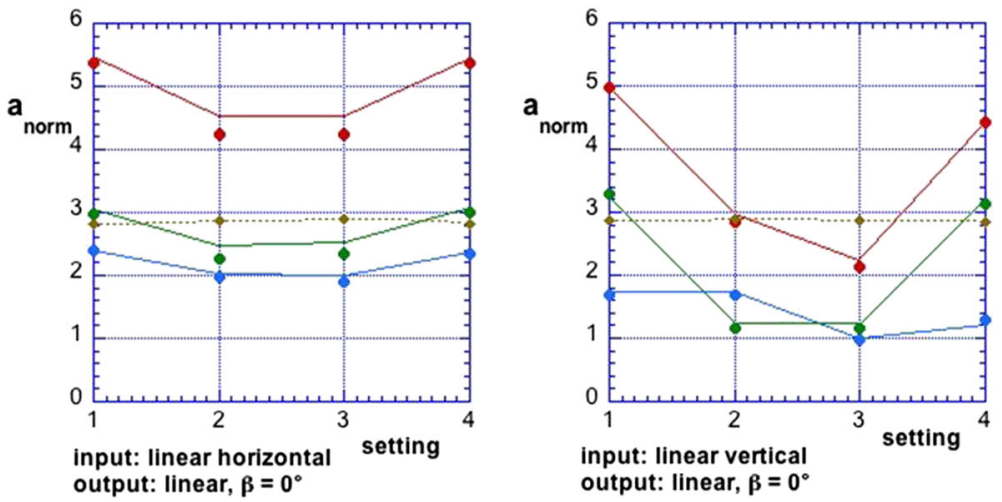

Fig. 9 Measured and calculated normalized losses for the four possible settings leading to a linear horizontal output polarization. Left, horizontal linear input; right, vertical linear input; dots, experimental data; lines, model

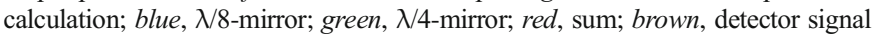



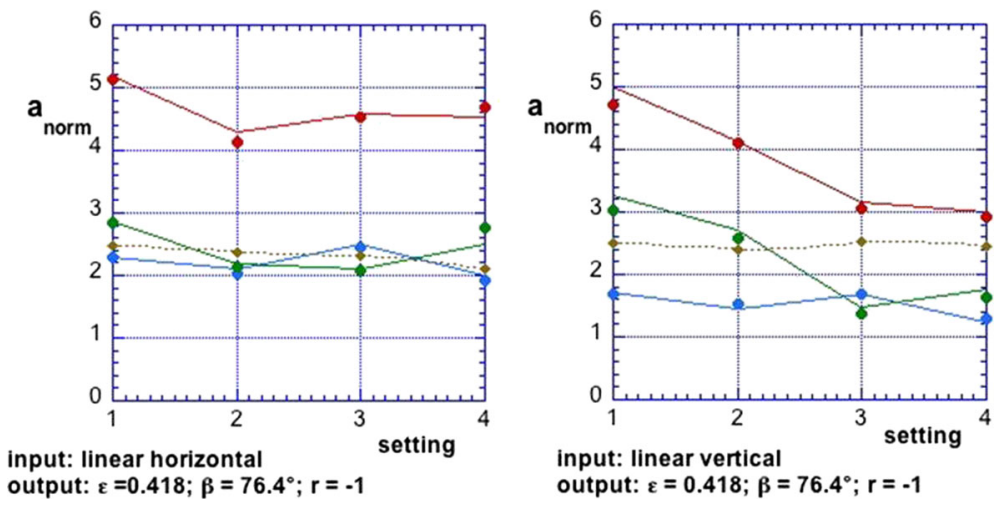

Fig. 10 Measured and calculated normalized losses for the four possible settings leading to an elliptical output polarization. Left, horizontal linear input; right, vertical linear input; dots, experimental data; lines, model calculation; blue, $\lambda / 8$-mirror; green, $\lambda / 4$-mirror; red, sum; brown, detector signal

too, we see a variation of the absorption coefficients in the range of 2.9 to 4.7 for the different possible settings.

We see a proper choice among the four possible mirror settings can greatly reduce the heat load on the polarizer mirrors and thus increase the power capability of the transmission line.

\section{Model Calculation}

As mentioned already in Chapter 2, the $\cos (2 \varphi)$ and $\cos (2 \alpha)$ dependencies of the losses suggest that they can be described as depending on the square of the incident field components. We therefore make an empirical Ansatz:

$$
a_{\text {norm }}(\alpha, \phi)=A \cdot E_{x}^{2}+B \cdot E_{z}^{2}+C \cdot H_{x}^{2}+D \cdot H_{z}^{2}
$$

considering only the tangential fields as defined for the mirror coordinate system shown in Fig. 5. We also make the assumption that a calculation based on infinite plane waves is a good approximation for the center part of the beam.

The relation between the incident fields in the waveguide coordinate system and the tangential fields in the mirror system is

$$
\begin{aligned}
& E_{x}=E_{0} \cos \alpha \sin \phi+\frac{1}{2} \sqrt{2} E_{0} \sin \alpha \cos \phi \\
& E_{z}=E_{0} \sin \alpha \sin \phi-\frac{1}{2} \sqrt{2} E_{0} \cos \alpha \cos \phi \\
& H_{x}=H_{0} \cos \alpha \cos \phi-\frac{1}{2} \sqrt{2} H_{0} \sin \alpha \sin \phi \\
& H_{z}=H_{0} \sin \alpha \cos \phi+\frac{1}{2} \sqrt{2} H_{0} \cos \alpha \sin \phi
\end{aligned}
$$

where $E_{0}$ and $H_{0}$ are the field amplitudes in the waveguide, and the factor $\sqrt{2} / 2$ is due to the $45^{\circ}$ incidence on the mirror. 
This relation simplifies for $\alpha=0^{\circ}$ or $90^{\circ}$ and for $\varphi=0^{\circ}$ or $90^{\circ}$. Inserting this into Eq. (8) and setting $E_{0}=H_{0}=1$, we get:

For $\alpha=0^{\circ}$ and $\varphi=0^{\circ}$ :

$$
0+\frac{1}{2} B+C+0=a_{\text {norm }, 0^{\circ}, 0^{\circ}}
$$

For $\alpha=0^{\circ}$ and $\varphi=90^{\circ}$ :

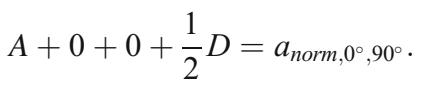

For $\alpha=90^{\circ}$ and $\varphi=0^{\circ}$ :

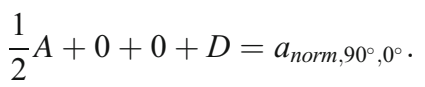

For $\alpha=90^{\circ}$ and $\varphi=90^{\circ}$ :

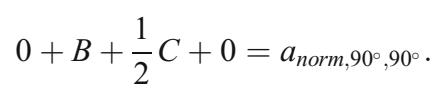

Thus, from our measurements of $a_{\text {norm }}$ at these $\alpha$ and $\varphi$ combinations, we can derive the loss coefficients A, B, C, and D. We have done this for the data of Fig. 6 with the result given in Table 1 .

Using the coefficients in Table 1 in Eq. (8), we can calculate the $\alpha$ and $\varphi$ dependencies of the losses of the respective mirrors. The results are shown in Figs. 4, 6, and 7 by the lines named model calculation. We see an astonishingly good agreement with this simple model. In Fig. 11, we show once more the calculated results of Figs. 4, 6, and 7 together with the detailed contributions of $E_{x}{ }^{2}, H_{x}{ }^{2}$, and $H_{z}{ }^{2}$. The contribution of $E_{z}{ }^{2}$ is not shown because it is in all cases negligibly small. This makes sense because $E_{z}$ must vanish along the ridges of the grooves and cannot excite a wave inside the grooves.

For the plane mirror, the result is clear: The surface current is proportional to the squared tangential magnetic field, which at $\varphi=0^{\circ}$ incidence has the full amplitude $\mathrm{H}_{0}$; while at $\varphi=90^{\circ}$, the tangential $\mathrm{x}$-component has only a magnitude of $1 / 2 \cdot \sqrt{2}$. $H_{0}$. And there is no $E_{x}$ contribution as there is no tangential electric field. The corrugated mirror which behaves somewhat similar to the plane mirror is the $\lambda / 8$ mirror with $\alpha=90^{\circ}$, but the $H_{x}$ and $H_{z}$ contributions are higher than in the plane mirror case and there is a small $E_{x}$ contribution. In the other cases, the $E_{x}$ contribution is considerably higher. All these components can excite a wave propagating in the grooves, thus increasing the losses with respect to the plane mirror.

Table 1 Loss coefficients for the plane and corrugated mirrors under test

\begin{tabular}{llll}
\hline & Plane mirror & $\lambda / 8$-mirror & $\lambda / 4$-mirror \\
\hline A & 0 & 0.60 & 2.83 \\
B & 0 & 0.01 & 0.01 \\
C & 1.41 & 2.01 & 2.45 \\
D & 1.41 & 2.25 & 16 \\
\hline
\end{tabular}



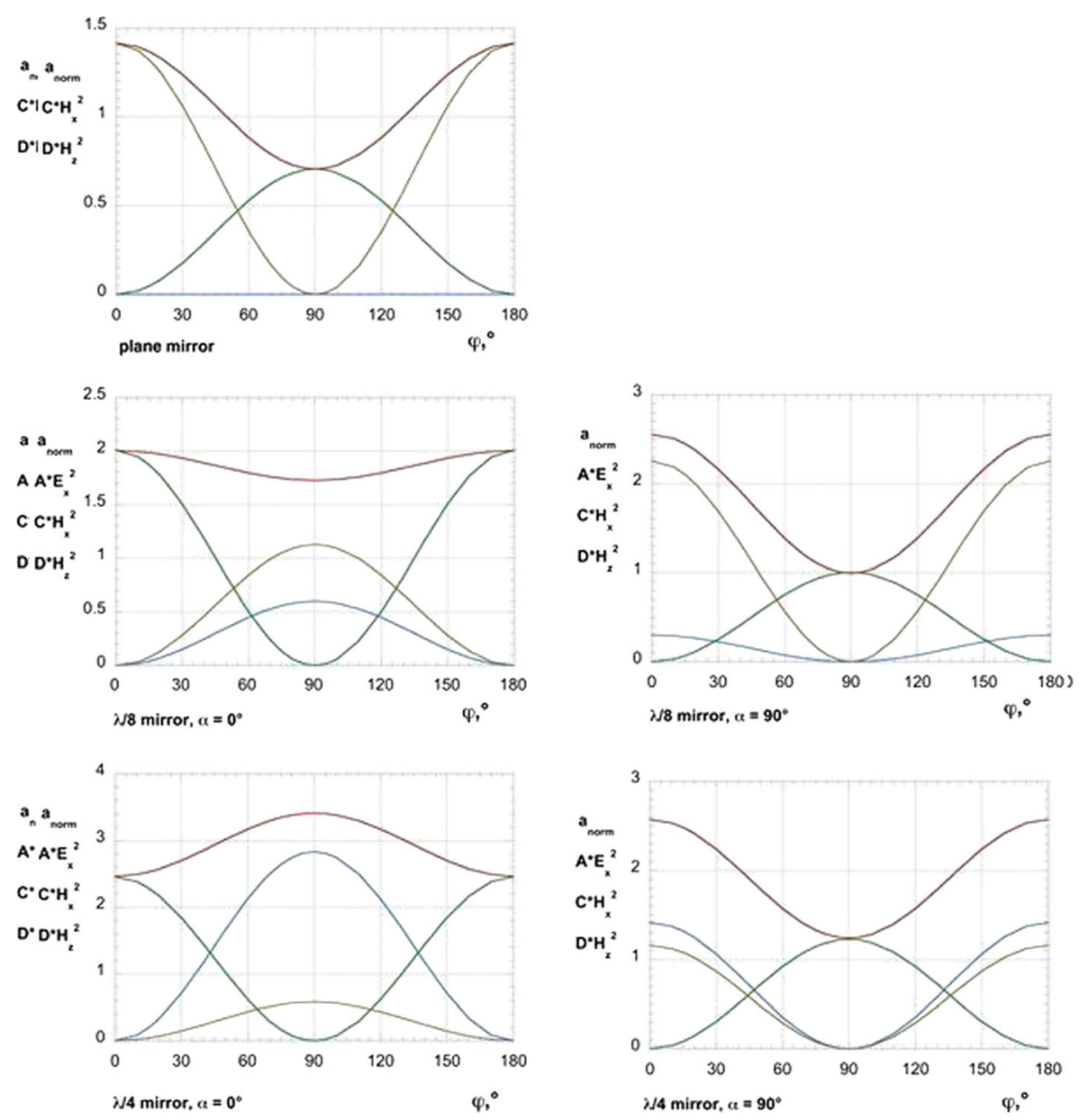

Fig. 11 Contributions of $\mathrm{A} \cdot \mathrm{E}_{\mathrm{x}}{ }^{2}$ (blue), $\mathrm{C} \cdot \mathrm{H}_{\mathrm{x}}{ }^{2}$ (green), and $\mathrm{D} \cdot \mathrm{H}_{\mathrm{z}}{ }^{2}$ (brown) to $a_{\text {norm }}$ (red) for a plane mirror (top), the $\lambda / 8$-mirror with $\alpha=0^{\circ}$ and $\alpha=90^{\circ}$ (center), and the $\lambda / 4$-mirror with $\alpha=0^{\circ}$ and $\alpha=90^{\circ}$ (bottom)

Our model can also be applied to the special cases of Chapter 3.3 with combinations of $\lambda / 8$ and $\lambda / 4$ mirrors. The results are also shown in Figs. 9 and 10 and reproduce the experimental data pretty well. The simple model given in Eq. (8) is thus well suited to calculate the losses of a pair of corrugated mirrors for any setting and for any geometrical arrangement, once the coefficients A, B, C, and $\mathrm{D}$ for the individual mirrors are determined. It can be integrated in a polarization matrix code as described in [9].

Based on this finding, we can now also calculate $2 \mathrm{D}$ plots of the losses depending on the rotation angles $\alpha$ of the $\lambda / 8$ and $\lambda / 4$ mirrors. In Fig. 12, we show, as an example, calculations of the output polarization for an input with horizontal linear polarization and the configuration given in Fig. 8. Shown are 2D plots of ellipticity $\varepsilon$, orientation $\beta$, rotation $r$, and losses $a_{\text {norm }}$. These figures are similar to the ones given in [9], but now including losses. The data points in these graphs show the possible settings for a scan 

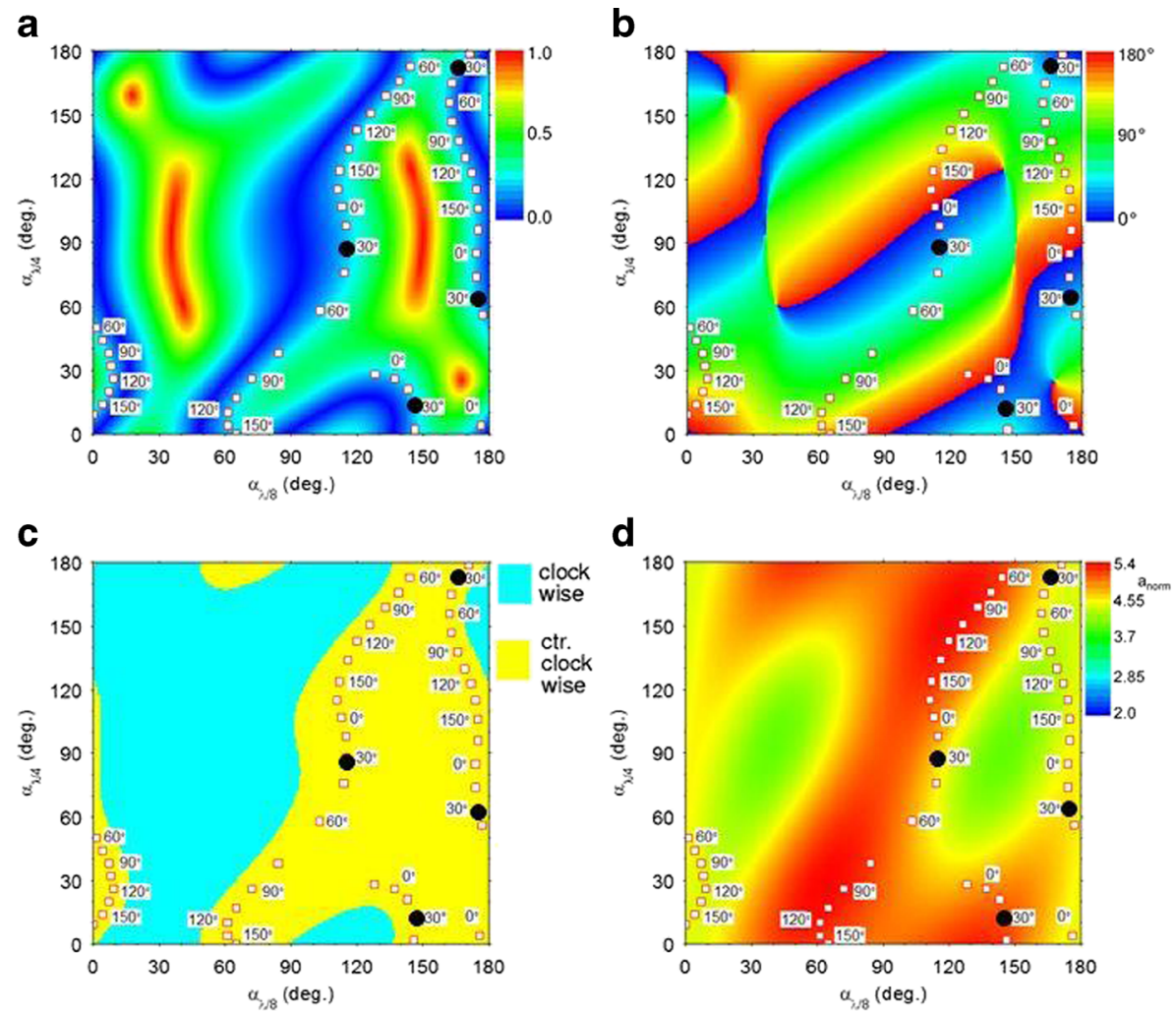

Fig. 12 2D plots of the output of a two-mirror polarizer fed by a horizontal linear input polarization. a Ellipticity $\varepsilon$. b Orientation $\beta$. c Rotation $r$. $\mathbf{d}$ Losses $a_{\text {norm }}$. The dots represent a scan of the orientation $\beta$ at constant $\varepsilon=0.2$ and $r=1$. As an example, the black dots highlight the four solutions for $\beta=30^{\circ}$

of the orientation $\beta$ from 0 to $180^{\circ}$ of the output polarization ellipse with $\varepsilon=0.2$ and rotation $r=+1$ (ctr. clockwise) with a horizontal linear input polarization. The dots represent solutions for the required output polarization with a cross-polarization content $<0.1 \%$ of the total power.

In Fig. 13, we show 2D plots for different ellipticities $\varepsilon$, the dots again representing a $\beta$-scan. We see that at low ellipticities of $\varepsilon=0$ and 0.2 , there are four solutions for the mirror settings at any value of $\beta$, whereas at the ellipticities $\varepsilon=0.4$ and 0.6 , we find for some $\beta$-values only two solutions. As an example, the black dots show the solutions for $\beta=60^{\circ}$.

In Fig. 14, we consider two cases with four solutions for which we show only plots of the losses. The data points are again for a scan of the orientation $\beta$ from $0^{\circ}$ to $180^{\circ}$, but for the ellipticities $\varepsilon=0$ (linear) and $\varepsilon=0.2$, and both for a linear input polarization either horizontal or vertical.

We see that the four possible settings, as shown in Fig. 14, to realize a wanted output polarization have quite different total loss, both for the linear and the elliptical output case. Lower loss can be obtained for a vertical input 

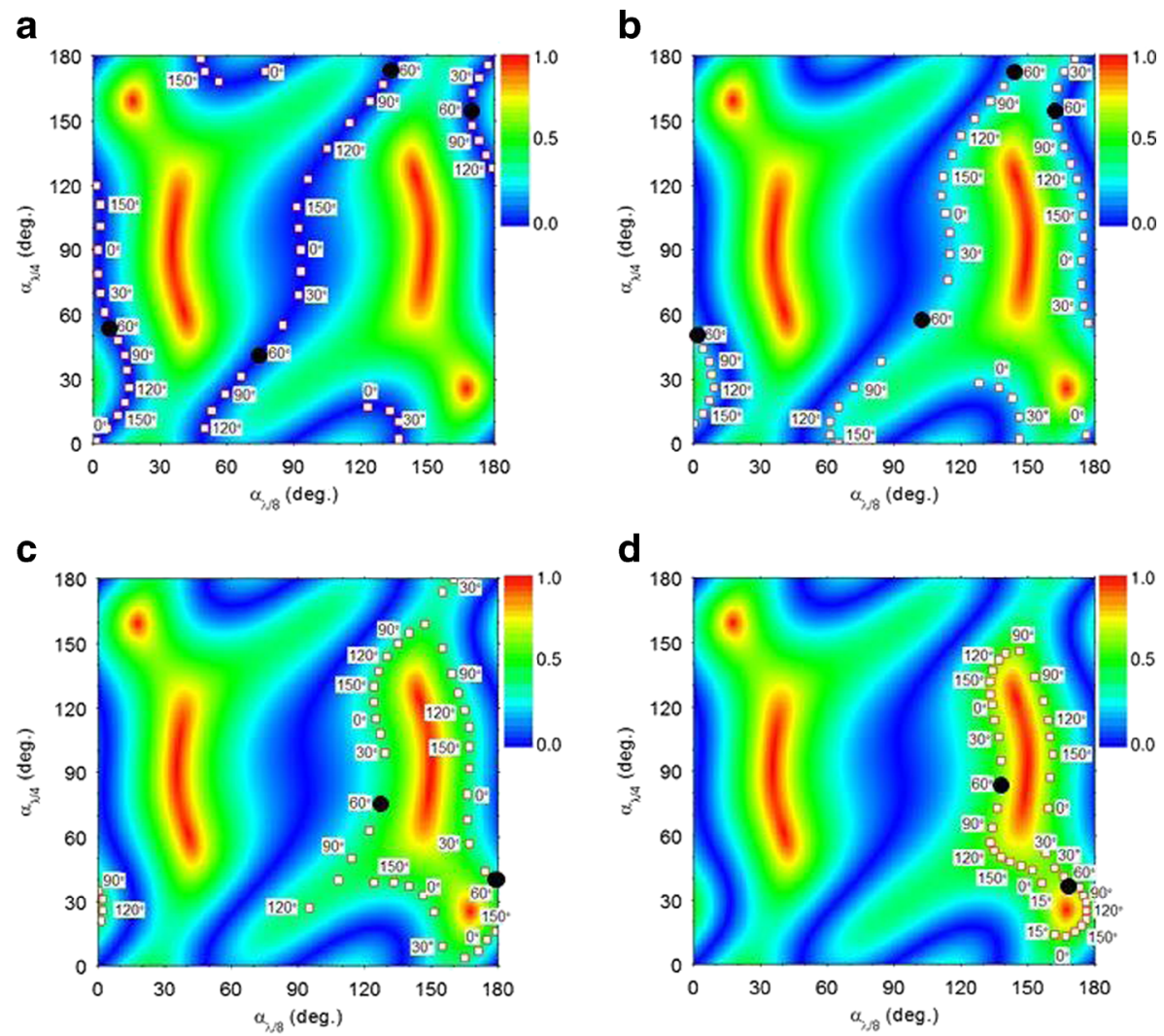

Fig. $132 \mathrm{D}$ plots of the calculated ellipticity at the output of a two-mirror polarizer fed by a horizontal linear input polarization. The dots represent a scan of the orientation $\beta$ at rotation $r=1$ for constant ellipticties of $\mathbf{a} \varepsilon=$ $0.2, \mathbf{b} \varepsilon=0.2$, $\mathbf{c} \varepsilon=0.4$, and $\mathbf{d} \varepsilon=0.6$. As an example, the black dots highlight the solutions for $\beta=60^{\circ}$

polarization ( $H$-plane incidence). This result is, however, true only for the polarizer arrangement, Fig. 8, as discussed here.

\section{Discussion}

Our model is not a theoretical one, rather an empirical one. The coefficients A, B, $\mathrm{C}$, and D for each mirror were obtained experimentally. Normalized to perpendicular incidence on a plane mirror they are valid for any material, not just stainless steel as used here. But they will depend on the corrugation parameters like profile shape, depth, and periodicity [7]. Nevertheless, they give a guideline how to choose a setting of the polarizer mirrors which leads to lower losses. A numerical code for polarizers like the one described in [6] should be able to calculate the coefficients A, B, C, and D where the calculation of only four special cases according to Eq. (8) is necessary. Experimentally, the coefficients can also be obtained at low power in a three-mirror-resonator setup as in [7]. In such a setup, only cases with linear 
a

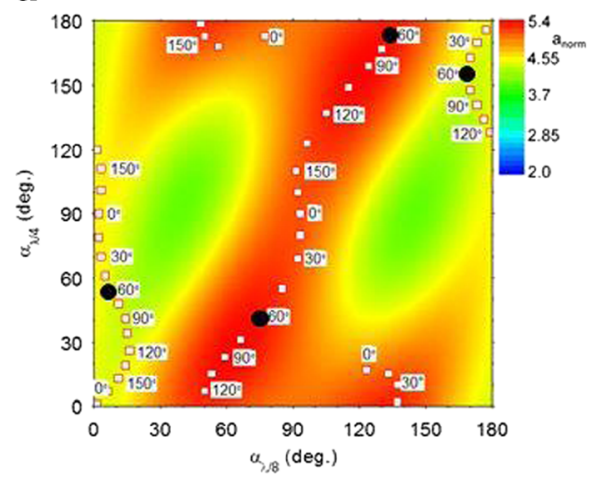

C

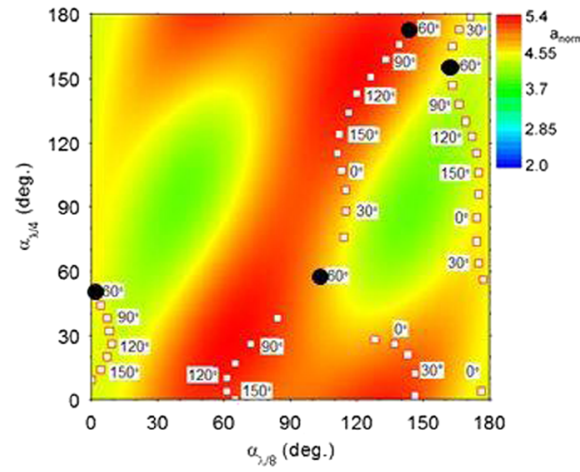

b

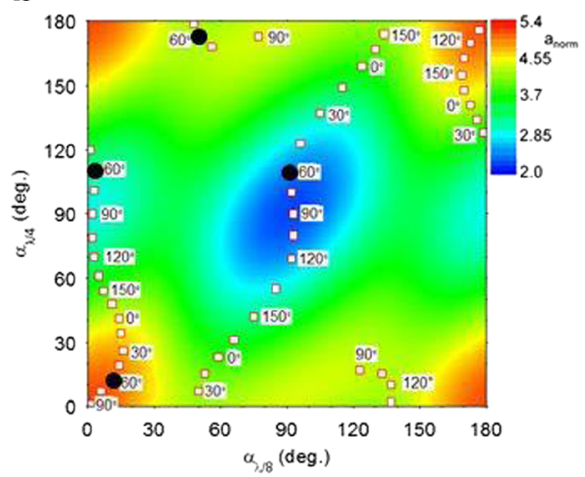

d

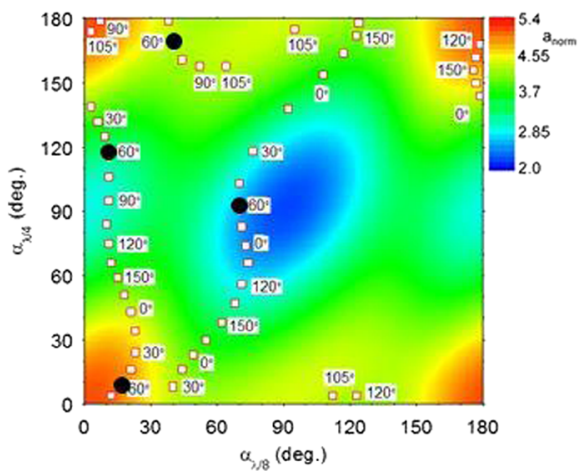

Fig. $142 \mathrm{D}$ plots of the total polarizer loss as function of the two-mirror settings $\alpha$. The data points show a scan of the orientation $\beta$ of the output polarization ellipse from $0^{\circ}$ to $180^{\circ}$ at constant ellipticity $\varepsilon$ and constant rotation $r$. As an example, the black dots highlight the four solutions for $\beta=60^{\circ}$. a Input, horizontal linear; output, $\varepsilon=0$, $r= \pm 1$ (lin. pol.). b Input, vertical linear; output, $\varepsilon=0, r= \pm 1$ (lin. pol.). c Input, horizontal linear; output, $\varepsilon=0.2$, $r=+1$ (ell. pol.). d Input, vertical linear; output, $\varepsilon=0.2, r=+1$ (ell. pol.)

polarization equal for input and output, and thus also only cases with parallel or perpendicular orientation of the grooves w.r.t., the E-field vector can be studied. These are just the cases required to determine the four parameters A, $\mathrm{B}, \mathrm{C}$, and D.

Applied to a universal polarizer consisting of two corrugated mirrors as done above the result for the total losses will also depend on the specific arrangement: We used here the arrangement in Fig. 8, but other arrangements where the two incidence planes are parallel or perpendicular to each other can also be treated with this model in the same way. One needs only to know the parameters A, B, $\mathrm{C}$, and $\mathrm{D}$ for the applied mirrors. In general, for the proper choice of the mirror settings, we need also to consider the changes of the polarization along the downstream transmission line including the launcher mirror. There is no doubt that in the case of a complex but fixed transmission line geometry, there is also an optimum position for the polarizer mirrors which can be found using this model. 
Acknowledgments We would like to thank our colleagues F. Monaco and H. Schütz for their valuable help in setting up and performing the high-power experiments and for proposing to use a PT-100 detector instead of a thermocouple. Open access funding provided by Max Planck Society.

Open Access This article is distributed under the terms of the Creative Commons Attribution 4.0 International License (http://creativecommons.org/licenses/by/4.0/), which permits unrestricted use, distribution, and reproduction in any medium, provided you give appropriate credit to the original author(s) and the source, provide a link to the Creative Commons license, and indicate if changes were made.

\section{References}

1. M.I. Petelin, Proc. First Intl. Workshop on Electron Cyclotron Resonance Heating Transmission Systems, Proc. First Intl. Workshop on Electron Cyclotron Resonance Heating Transmission Systems (Cocoa Beach, Florida, 1990).

2. J.L. Doane et al., Polarizer miter bends for high-power microwave transmission: Ohmic loss and cooling, Fusion Engineering and Design, Vol. 102 (2016), 99-107.

3. Y. Kashiwa et al., Study of Ohmic loss of high power polarizers at $170 \mathrm{GHz}$ for ITER, Fusion Engineering and Design, Vol. 81 (2006), 2249-2256.

4. D. Wagner et al., New frequency step-tunable ECRH System for ASDEX Upgrade, International Journal of Infrared and Millimeter Waves, Vol. 27 (2006), 173-182.

5. V. Erckmann, P. Brand, H. Braune, G. Dammertz, G. Gantenbein, W. Kasparek, H.P. Laqua, H. Maassberg, N.B. Marushchenko, G. Michel et al., Electron Cyclotron Heating for W7-X: Physics and Technology, Fusion Science and Technology 52, 291 (2007)

6. B. Plaum et al., Numerical Calculation of Reflection Characteristics of Grooved Surfaces with a 2D FDTD Algorithm, Journal of Infrared, Millimeter and Terahertz Waves, Vol. 32 (2011), 482-495.

7. W. Kasparek et al., Measurement of ohmic loss of metallic reflectors at $140 \mathrm{GHz}$ by a 3-mirror resonator technique, Int. J. Infrared Millimeter Waves, Vol. 22 (2001), 1695-1707.

8. D. Wagner et al, Status, Operation and Extension of the ECRH System at ASDEX Upgrade, International Journal of Infrared, Millimeter and Terahertz Waves, Vol. 37 (2016), 45-54.

9. D. Wagner, F. Leuterer, Broadband polarizers for high power multi-frequency ECRH systems, Journal of Infrared, Millimeter and Terahertz Waves, Vol. 26 (2005), 163-172.

10. M.K. Thumm, W. Kasparek, Passive High-Power Microwave Components, IEEE Trans. on Plasma Science, Vol. 30, No. 3 (2002), $755-786$. 